\title{
Callosal disconnection syndrome and knowledge of the body: a case of left hand isolation from the body schema with names
}

\author{
Toyoko Nagumo, Atsushi Yamadori
}

\begin{abstract}
A patient is described who presented with a disturbance of body cognition confined to the left side of the body. She showed difficulties in naming the left fingers and in moving the named left fingers. She also showed great difficulty in pointing to named parts of the body with her left hand. Earlier in the course of the disease she showed a personification phenomenon of the left hand. Brain MRI showed involvement of the entire corpus callosum, probably due to occlusion of a branch of the anterior cerebral artery. It is speculated that this syndrome is caused by disconnection of the right hemisphere from the left hemispheric body schema.
\end{abstract}

(F Neurol Neurosurg Psychiatry 1995;59:548-551)

Keywords: callosal disconnection syndrome, body schema, autotopagnosia, tactokinesthetic two way anomia.

We encountered a patient who showed an array of disconnection syndromes after an infarction of the corpus callosum. We have already reported the left sided avoiding reaction of this patient and discussed the symptom in terms of possible disconnection of the asymmetrically organised manual spatial function. ${ }^{1}$ Other intriguing symptoms of this patient were a left sided alien hand $\operatorname{sign}^{23}$ in the early course of the disease and persistent difficulty in identifying body parts with the left hand. To our knowledge, reports of unilateral autotopagnosia associated with the callosal disconnection syndrome have not appeared in the medical literature.

We studied the symptoms in detail and speculate on an underlying mechanism in terms of lateralisation of a body recognising system - that is, a body schema.

\section{Case presentation}

The details of this 56 year old right handed housewife have already been reported. ${ }^{1}$ In March 1988 she had a cerebral infarction, most likely a thrombosis of a branch of the left anterior cerebral artery. Neuroimaging studies including CT and MRI confirmed an ischaemic lesion confined to the left half of the corpus callosum from the genu through the entire length of the body (fig 1).
Subsequently, she showed various typical disconnection syndromes including alien left hand sign, diagonistic apraxia, self grasping by the left hand, left hand tactile anomia, left hand agraphia, left ideomotor apraxia, left sided motor initiation difficulty, left sided crossed avoiding, ${ }^{4}$ and left unilateral neglect when drawing with the right hand. ${ }^{5}$ This left unilateral spatial neglect was never seen with tasks involving the left hand. General behavioural neglect toward the left was not seen in daily activities.

On 20 December 1988 she was alert and cooperative. Cranial nerves were normal including the visual fields. Movements of the left upper and lower limbs were clumsy in finger to nose and heel to shin tests, but no clear weakness or ataxia was present. Muscle tone was normal. Deep tendon reflexes were increased bilaterally but no pathological reflexes were detected. Superficial sensation was normal. Joint position sense was impaired in the fingers of the left hand. Vibration was slightly decreased bilaterally. For stereognosis, the right hand was normal and the left hand

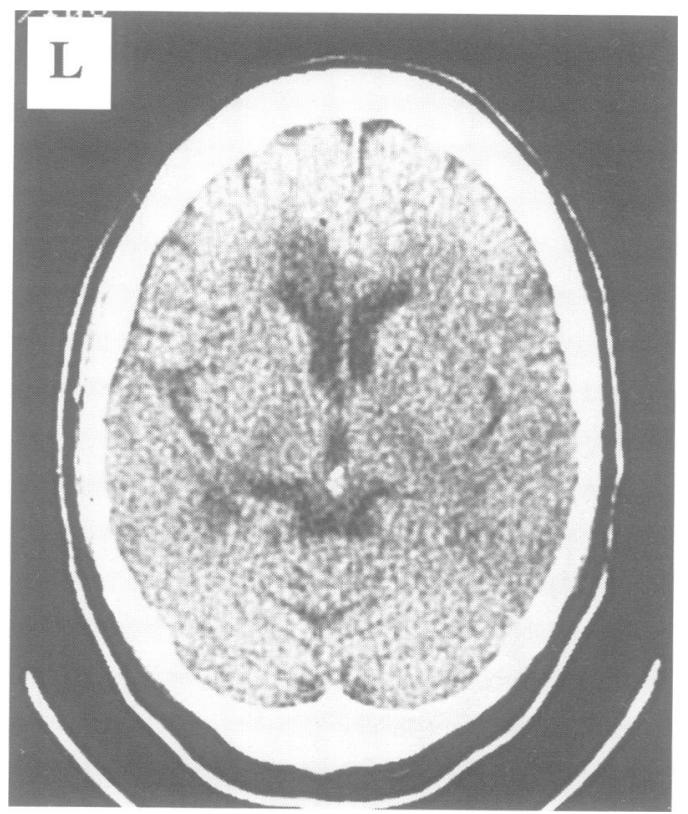

Figure 1 CT of the brain showing circumscribed low absorption areas in the corpus callosum and the adjacent medial area of the left frontal lobe consistent with a diagnosis of infarction of the left anterior cerebral artery. 
was correct for $80 \%$ in non-verbal response tasks.

No appreciable change was detected in the neurological signs at a re-examination three years after the onset. In this examination we tested for the first time left-right transfer of tactile localisation and finger pattern. Transfer from the right to the left often elicited stiffness of the left hand. Even when the left-right transfer was feasible, it ended in errors.

Brain CT taken four years after the onset showed an advance of the cortical atrophy in the left frontal area. The anterior horn of the left hemisphere was enlarged and the damage of the corpus callosum at this level was clear.

\section{Studies on body identification} PERSONIFICATION

For three months after the onset she showed a pronounced personification phenomena ${ }^{6}$ of the left, non-paralysed, hand. She often behaved as if the left hand were a real person. The personification usually occurred when she could not control her left hand voluntarily. Here is an example:

When she was being tested for apraxia, the left hand grasped the right hand performing a task. She tried to let the left hand go to no avail. Then she started talking towards the left hand, "Grandma, would you please let me go. You are warm, but my hand is sweating and uneasy."

\section{IDENTIFICATION OF THE PATIENT'S OWN} BODY

Based on the method described by Benton ${ }^{7}$, extensive tests of body identification were performed over a two year period one year after the onset. During this period no personification phenomena were seen. The table summarises the test results.

For naming of fingers three methods were employed, visual naming and two non-visual methods of naming a finger touched by an examiner with the patient blindfolded and with the hand behind the patient's back. For comprehension of finger names, two methods were used: pointing to a named finger with the contralateral hand and moving a named finger. For non-verbal identification of fingers the patient was asked to move a touched finger. For identification of body parts the patient was asked to name a touched part, point to a named part, and to point to a touched part

The patient's performance was perfect for visual naming of the fingers and the body. Non-visual naming, and naming of the body parts and the right fingers were perfect; however, the patient performed very poorly for non-visual naming of the left fingers. The patient performed accurately for pointing to a named left finger with the right hand with the eyes open or closed, but was poor in pointing to the right fingers with the left hand, being much worse when blindfolded. Pointing to a named body part with the right hand was perfect (fig 2), but it was poor with the left hand both with the eyes closed and open.

Identification of a named finger by moving it was perfect for the right hand with the eyes

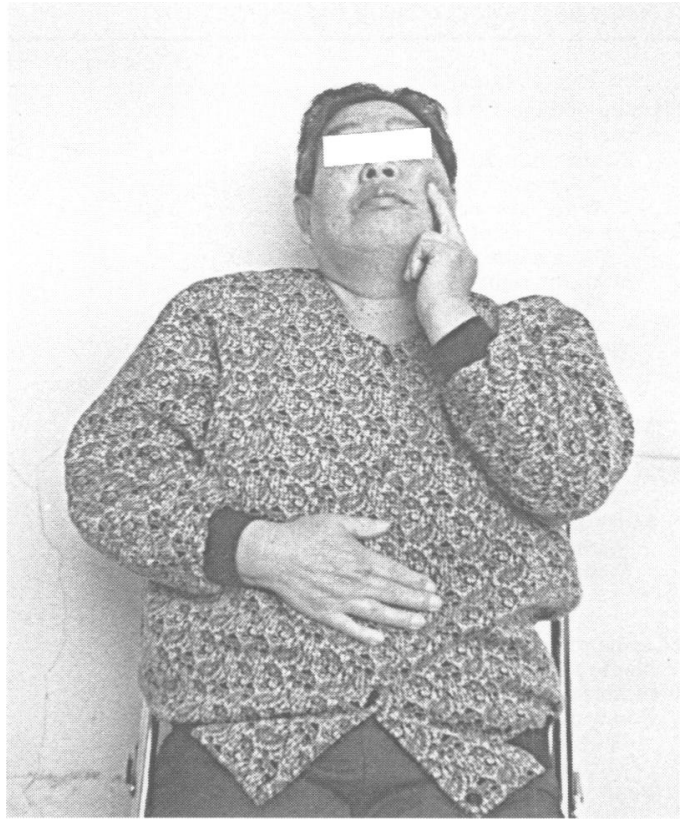

Figure 2 Identification of body parts: patient was asked to point to her abdomen. The right hand always responded accurately.

open, the eyes closed, and the hand behind the body. It was remarkably poor for the left hand, being much worse when blindfolded. With the non-verbal identification task of moving a touched finger, the left fingers responded correctly in both visual and non-visual conditions. Body part identification by pointing to a touched part was fairly good with the left hand, even when non-visual.

\section{IDENTIFICATION OF THE EXAMINER'S BODY}

Visual naming of the examiner's body parts including the fingers was perfect. Pointing to the named parts of the examiner's body was also without error if performed with the right hand. Correct pointing rate with the left hand, however, fell to $79 \%$.

\section{POINTING TO OBJECTS}

Finally we tested pointing ability of the left hand with objects. Five objects were placed on a desk, the patient pointed to a named object when able to see and when blindfolded. Under the visual condition, the patient's response was good. With 10 trials (50 pointings) she was $97 \%$ correct. Under the non-visual condition, the response became slow and poor, but the performance improved after she memorised the location of objects. With 10 trials (50 pointings) she was $67 \%$ correct. We tested visual pointing capacity of her left hand for six objects, placed on her forehead, ear, shoulder, breast, navel, and knee. ${ }^{8}$ With the total of five sessions (30 pointings) she was $80 \%$ correct. When the task was switched to pointing to a named body part she was correct in only $37 \%$ of 30 pointings. For both tests the performance of the right hand was normal.

\section{OTHER TESTS RELATED TO THE BODY IMAGE} We asked 14 verbal questions describing a relation of the various body parts. For 
Naming and identification of body parts

\begin{tabular}{|c|c|c|}
\hline & Right fingers & Left fingers \\
\hline Visual naming & 100 & 100 \\
\hline \multicolumn{3}{|l|}{ Name a touched finger } \\
\hline With eyes closed & 100 & 32 \\
\hline With hand behind body & 100 & 13 \\
\hline \multicolumn{3}{|l|}{ Identification of fingers: } \\
\hline \multicolumn{3}{|l|}{ Point to a named finger } \\
\hline \multicolumn{3}{|l|}{ With the right hand } \\
\hline $\begin{array}{l}\text { With eyes open } \\
\text { With eves closed }\end{array}$ & & 100 \\
\hline $\begin{array}{l}\text { With eyes closed } \\
\text { With the left }\end{array}$ & & 97 \\
\hline \multicolumn{3}{|l|}{ With the left hand } \\
\hline With eyes open & 64 & \\
\hline With eyes closed & 26 & \\
\hline \multicolumn{3}{|l|}{ Move a named finger } \\
\hline With eyes open & 100 & 52 \\
\hline With eyes closed & 100 & 24 \\
\hline With hand behind body & 100 & 0 \\
\hline \multicolumn{3}{|l|}{ Move a touched finger } \\
\hline \multirow{3}{*}{$\begin{array}{l}\text { With eyes open } \\
\text { With eyes closed }\end{array}$} & 100 & 98 \\
\hline & 100 & 98 \\
\hline & Eyes open & Eyes closed \\
\hline Naming of body parts (\% correct): & & \\
\hline Name a touched part & 100 & 95 \\
\hline \multicolumn{3}{|l|}{ Identification of body parts } \\
\hline \multicolumn{3}{|l|}{ Point to a named part } \\
\hline With the right hand & 99 & 100 \\
\hline & 41 & 30 \\
\hline \multicolumn{3}{|l|}{ Point to a touched part } \\
\hline With the right hand & 100 & 100 \\
\hline With the left hand & 97 & 85 \\
\hline
\end{tabular}

Body parts included head, ear, forehead, eye, cheek, nose, mouth, chin, neck, shoulder, chest,

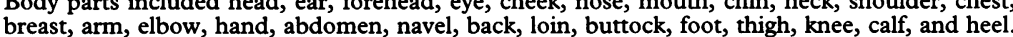
The number of tests were: finger test; 5 fingers times 10 for each; body part naming; 30 sites The number of tests were: finger test; 5 fingers times 10 for each; body part naming; 30
times 3 ; body parts pointing; 30 sites times 5; right and left hands were tested alternately.

instance, we asked the correctness of the following statement: "The mouth is situated above the eyes." She scored 27 points out of expected 28. We also asked her to draw a human figure. Her drawing was clumsy. The lengths of the arms and legs were short relative to the trunk, but the spatial relation between various body parts was well preserved. According to MacDonald's scoring method ${ }^{9}$ the right hand's score was 8 of 10 and the left hand's score was 7 .

\section{Discussion}

The present case presented a unique combination of signs confined to the left hand. Essentially there were two core symptoms. One was the difficulty of left finger identification. She could neither name the left fingers nor move the named left fingers when vision was excluded. Under visual conditions these tasks were perfectly conducted. The other was the difficulty of pointing to the named body parts with the left hand. The difficulty covered the whole body including the right fingers. Again the right hand showed no difficulty.

Autotopagnosia was first described by Pick in 1909 and defined as patients' inability to localise and name the parts of their own body correctly. ${ }^{10}$ Finger agnosia, described by Gerstmann in $1924,{ }^{11}$ belongs to this category of autotopagnosia. Typical patients would not be able to name or move the parts of their own bodies touched by an examiner, or move or indicate the parts named by the examiner. The disorder was always bilateral. Drawing of a human figure may be abnormal.

The present case can be placed in this broad category of autotopagnosia but with exceptional features. Thus we have a case of tactokinesthetic autotopagnosia confined to the left fingers associated with left hand disorientation for named body parts.

Tactokinaesthetic left finger agnosia can be explained in terms of modality specific as well as category specific two way anomia. Two way anomia was first described by Geschwind and Fusillo in the colour domain. ${ }^{12}$ Their patient had difficulty naming colours and pointing to them but preserved the ability to sort colours according to hues, match colours, and recall colour names in response to verbal questions. They explained the symptoms as a disconnection of the linguistic system from the right hemispheric colour system because of combined damage to the left occipital visual system and the corpus callosum, through which the colour information of the right hemisphere passes to the left language area. The identical argument is feasible in the present case. Suppose a reference system for the location and the names of the body-call it the body image with names-is lateralised in the left hemisphere. If this body image with names was separated from the right hemisphere by an appropriately placed callosal lesion, anomia as well as comprehension difficulty for the names of parts of left side of the body would inevitably result. Tactospatial information from the left finger area must be first processed in the contralateral right hemisphere and subsequently have to pass to the left hemisphere for reference for a body part identification and a name. When a name of the left fingers was provided, the left hemispheric body image with names was activated, but could not transmit this exact localising information to an appropriate area of the right motor area. Axial body information may reach the left hemisphere ipsilaterally resulting in no difficulty in identification. As the right somesthetic processing area is essentially intact, tactile localisation of a touched finger by moving the same finger should be possible.

How can we reconcile the peculiar left hand difficulty of pointing to the named body parts bilaterally with this two way anomia theory? When given a name, the left hand showed disorientation and could not arrive at a correct destination. But the performance was better when visual than when blindfolded. When given tactile information (touch), the same hand localised the point fairly well under both conditions. This left hand performance could not be due to apraxia or visuomotor ataxia, as the spatial reaching at an object outside the patient's own body was good as shown by her ability in pointing to the body parts of the examiner and pointing to objects even on her own body. The left hand, under right hemispheric control, was unable to relate its spatiomotor information with the left hemispheric body image with names through the corpus callosum.

This theory is consistent with previous ones. Gerstmann, ${ }^{13}$ for instance, argued that the body schema should be lateralised to the left hemisphere. From a study of "pure" autotopagnosia in which verbal and non-verbal identification of body parts is impaired, Semennza and Goodglass postulated the exis- 
tence of a conceptual representation of body parts and their spatial and functional interrelations in the left hemisphere. ${ }^{14}$ Based on studies of anosognosia Yamadori also argued that the body schema is still a relevant neurological concept in interpreting symptoms related to difficulty in identifying parts of the body and is most likely to be lateralised in the left hemisphere. ${ }^{15}$ The present case seems to reinforce these arguments.

Was her left alien hand and personification episode consistent with this callosal disconnection theory of the body image with names? After Yamadori ${ }^{15}$ an argument can be presented. In the acute phase with a widespread callosal disconnection, the body schema principally organised in the left hemisphere would be thrown into imbalance and tend to be activated. The activation of the body schema would mean an autonomous activity of the body schema even in the absence of supporting information, in this particular case somesthetic information from the right hemisphere concerning the left half of the body. The autonomous body schema activation in the left hemisphere would generate an experience of a perfect body image. A sudden movement of the left hand driven by the disconnected right hemisphere would be perceived and appreciated as a movement. But it is disconnected from a feeling of the patient's having a complete body. A conscious left hemisphere ${ }^{16}$ would very likely interpret this hand movement as something outside its own body, resulting in alien hand sign.

Some might argue that the phenomenon could be interpreted as a partial symptom of the left sided neglect. It was true the patient showed left unilateral neglect with the right hand ${ }^{5}$, but not with the left hand. No spatial inattention toward the left side was seen behaviourally. If there was a problem, it was toward the right. ${ }^{1}$ Thus it is difficult to interpret the symptoms in terms of left side neglect. Even the personification phenomenon seen in the present case was different from the similar phenomenon often seen in association with anosognosia. ${ }^{6}$ The first is seen in response to a moving left hand, whereas the second is seen in association with a paralysed left hand.

We thank Dr Yoshiaki Soma, Dr Takeo Kuwabara at the Niigata University, Dr Yokichi Hayashi, and Ms Mari Hoshino for help in preparation of the manuscript. We also thank Dr Masakazu Ito, Director of Niigata Prefectural Muikamachi Hospital for supporting our study. Last but not least are thanks Hospital for supporting our study. Last but not least are thanks
to the patient, whose willing cooperation made this study to the patic
possible.

1 Nagumo T, Yamadori A, Soma Y, Kayamori R, Ito $M$. Crossed avoiding reaction: a disturbance of the manual spatial function. $f$ Neurol Neurosurg Psychiatry 1993;56: $552-5$.

2 Bogen JE. The callosal syndrome. In: Heilman KM, Valenstein E, eds. Clinical Neuropsychology. New York: Oxford University Press, 1979:308-59.

3 Feinberg TE, Schindler RJ, Flanagan NG, Haber LD. Two alien hand syndromes. Neurology 1992;42:19-24.

4 Lechevalier B, Andersson JC, Morin P. Hemispheric disconnection syndrome with a "crossed avoiding" reaction in a case of Marchiafava-Bignami disease. $\mathcal{f}$ Neurol Neurosurg Psychiatry 1977;40:483-97.

5 Kashiwagi A, Kashiwagi T, Nishikawa T, Tanabe $H$, Okuda J. Hemispatial neglect in a patient with callosal infarction. Brain 1990;113:1005-23.

6 Critchley M. The parietal lobes. New York: Hafner Press, 1953.

7 Benton AL. Right-left discrimination and finger localization. New York: Harper, 1959.

8 Sirigu A, Grafman J, Bressler K, Sunderland T. Multiple representations contribute to body knowledge processing. Brain 1991;114:629-42.

9 MacDonald J. An investigation of body scheme in adults with cerebral vascular accident. Am F Occup Ther 1960; 14:72-9.

10 Frederiks JAM. Disorders of the body schema. In: Frederiks JAM, ed. Handbook of clinical neurology. Vol 1(45): clinical neuropsychology. Amsterdam: Elsevier Science Publishers, 1985;373-404.

11 Gerstmann J: Fingeragnosie. Eine umschriebene Storung der Orientierung am eigenen Korper. Wien Klin Wochenschr 1924; 37:1010-12.

12 Geschwind N, Fusillo M. Color-naming defects in association with alexia. Arch Neurol 1965;15: 137-46.

13 Gerstmann J. Problem of imperception of disease and of impaired body territories with organic lesions. Arch Neurol 1942;48:890-913.

14 Semenza $C$, Goodglass $H$. Localization of body parts in brain injured subjects. Neuropsychologia 1985;23:161-75. 15 Yamadori A. Anosognosia. Neurol Med (Tokyo) 1989;30: 364-9. (In Japanese.)

16 Sperry RW, Gazzaniga MS, Bogen JE. Interhemispheric relationships: the neocortical commissures; syndromes of hemispheric disconnection. In: Vinken PJ, Bruyn GW, eds. Handbook of clinical neurology. Vol 4. Amsterdam: North-Holland, 1969;273-90. 